\title{
ON MONOTHETIC SEMIGROUPS 1
}

R. J. KOCH

By semigroup we shall mean a Hausdorff space together with a continuous associative multiplication. The study of monothetic semigroups has been initiated independently by several authors; most of the known results involve some form of compactness. We repeat here some of these known results for the sake of completeness. Among the results we establish is the fact that a compact monothetic semigroup with two distinct generators is a group. Some results are given on translates of compact sets in semigroups. Finally we establish analogs for semigroups of Weil's theorem on monothetic groups.

We use $S$ throughout to denote a semigroup, and follow the terminology of Clifford [1]. In particular a unit is a two-sided identity element and $K$ is the kernel (=minimal ideal) of $S$ (if it is not empty). For $a \in S$ we let $\Gamma(a)$ denote the closure of the set of positive powers of $a$. We say $S$ is monothetic if for some $a \in S, \Gamma(a)=S$. In such a case we say that $a$ is a generator of $S$. Note that if $S$ is monothetic then it is commutative. In the group case it is customary to use both positive and negative powers to define monotheticity [5]. It can be seen that the two notions agree in a compact group. To eliminate confusion we may for emphasis write monothetic $(+)$ or monothetic $( \pm)$. For the terminology of nets we follow Kelley [6]. Compactness is used in the sense of bicompactness.

It is a pleasure to record our obligations to Professor A. D. Wallace.

The following basic result on monothetic semigroups is due to Numakura [2], Peck [10], and the author independently; there is also a generalization due to Wallace [12].

TheOREM 1. Let $S$ be compact and monothetic with generator a. Then the cluster points of the net (sequence) $\left\{a^{n}\right\}$ form a group $K(a)$, and $K(a)$ is the minimal ideal of $S$.

We omit the proof. Theorem 1 yields immediately the fact that a compact semigroup contains an idempotent.

Corollary 1. A monothetic semigroup with unit is either a finite group or is dense in itself.

Proof. Let $a$ be a generator of $S$ and denote the unit by 1 . In case

Received by the editors March 28, 1955.

1 A portion of this work was done under Contract N7-ONR-434 Task order III, Office of Naval Research. 
there are integers $p$ and $q$ with $a^{p}=a^{q}$ we conclude from the theorem that $S$ is a finite group. In the other case if some element $x \in S$ is an isolated point then $x=a^{p}$ for some integer $p$. Since $\left\{a^{n}\right\}$ clusters at 1 , $\left\{a^{p+n}\right\}$ clusters at $a^{p}=x$ and the result follows.

COROLlaRy 2. Let $S$ be compact and monothetic with generator a. If a is not an isolated point, then $S$ is a (topological) group.

Proof. If $a$ is not an isolated point, then $\left\{a^{n}\right\}$ clusters at $a$, hence by the theorem $S$ is a group. The fact that a compact group is topological is contained in $[3 ; 11]$.

CoRollary 3. Let $S$ be compact and monothetic with two distinct generators; then $S$ is a (topological) group.

Proof. Let $a$ and $b$ be distinct generators of $S$, and denote by $A$ and $B$ the sets of positive powers of $a$ and of $b$ respectively. The proof is divided into two cases, according as $b \in A$ or $b \notin A$. Suppose $b \in A$; if $a \in B$ then there are positive integers $m$ and $n$ greater than 1 with $b=a^{n}$ and $a=b^{m}$. Hence $a=a^{m n}$ where $m n>1$, and it follows from the theorem that $S$ is a topological group. If $a \notin B$ then $a$ is not an isolated point, and the result follows from Corollary 2. Now suppose $b \notin A$; as in the preceding argument we again conclude that $S$ is a group.

The foregoing ideas find application in a pair of dual theorems which express a kind of continuity for translates of compact sets in a semigroup. We say that $S$ acts on a space $X$ if there is a continuous function $f: S \times X \rightarrow X$ (notation $f(s, x)=s(x)$ ) satisfying $\left(s s^{\prime}\right)(x)$ $=s\left(s^{\prime}(x)\right)$ for $s, s^{\prime} \in S, x \in X$. We assume $X$ is a $T_{1}$-space.

Theorem 2. Let $S$ act on $X$, let $A$ be a compact subset of $X$, and let $\left\{p_{\alpha}\right\}$ be a net in $S$ such that $\beta>\alpha$ implies $p_{\beta}(A) \subset p_{\alpha}(A)$. Then $\bigcap_{\alpha} p_{\alpha}(A)$ $=p(A)$ for any cluster point $p$ of the net $\left\{p_{\alpha}\right\}$.

Proof. Choose $x \in p(A)$; then for some $a \in A, x=p(a)$. Suppose $p(a) \notin \bigcap_{\alpha} p_{\alpha}(A)$; then for some $\beta, p(a) \notin p_{\beta}(A)$. Now $p_{\beta}(A)$ is compact so there is an open set $O$ about $p$ such that $O(a) \cap p_{\beta}(A)=\varnothing$. Hence there is $\gamma$ such that $\gamma>\beta$ and $p_{\gamma} \in O$. Also $p_{\gamma}(A) \subset p_{\beta}(A)$ so that $O(a) \cap p_{\gamma}(A)=\varnothing$. Therefore $p_{\gamma}(a) \notin p_{\gamma}(A)$, a contradiction. Now choose $x \in \bigcap_{\alpha} p_{\alpha}(A)$ and suppose $x \notin p(A)$. Using the compactness of $A$ we produce an open $V$ about $p$ with $x \in V(A)$. For some $\beta, p_{\beta} \in V$; hence $x \notin p_{\beta}(A)$, a contradiction.

Corollary 1. Let $S$ be compact, $a \in S$. Then $\cap_{n} S a^{n}=S e$ where $e^{2}=e \in \Gamma(a)$. 
The proof is immediate. The corollary is implicit in Numakura [8].

The following theorem is dual to Theorem 2.

Theorem 3. Let $S$ act on $X$, let $A$ be a compact subset of $X$, and let $\left\{p_{\alpha}\right\}$ be a net in $S$ such that $\beta>\alpha$ implies $p_{\beta}(A) \supset p_{\alpha}(A)$. Then $\left[\cup_{\alpha} p_{\alpha}(A)\right]^{*}=p(A)$ for any cluster point $p$ of the net $\left\{p_{\alpha}\right\}$.

Proof. Let $x \in\left[\mathrm{U}_{\alpha} p_{\alpha}(A)\right]^{*}$ and suppose $x \notin p(A)$. Then there are open sets $O$ about $x$ and $V$ about $p$ such that $O \cap V(A)=\varnothing$. Now $O \cap p_{\beta}(A) \neq \varnothing$ for some $\beta$, and there is $\gamma>\beta$ such that $p_{\gamma} \in V$. Hence $\varnothing \neq O \cap p_{\beta}(A) \subset O \cap p_{\gamma}(A) \subset O \cap V(A)$, a contradiction. Now let $p(a)$ $\in p(A)$ and suppose $p(a) \notin\left[\bigcup_{\alpha} p_{\alpha}(A)\right]^{*}$. Then there is an open $V$ containing $p$ such that $V(a) \cap \bigcup_{\alpha} p_{\alpha}(A)=\varnothing$. Hence there is $\beta$ such that $p_{\beta} \in V$, and $p_{\beta}(a) \in V(a) \subset X \backslash p_{\beta}(A)$, a contradiction.

The following corollary, which has been referred to as Wallace's swelling lemma, was proved in [12] using Theorem 2. We give a shorter proof based on Theorem 3.

Corollary 1. Let $A$ be a compact subset of $X$, let $p \in S$ with $\Gamma(p)$ compact and $p(A) \supset A$; then $p(A)=A$.

Proof. Since $p(A) \supset A, p^{n}(A) \supset \cdots \supset p(A) \supset A$ for each positive integer $n$. Hence $e(A) \supset p(A) \supset A$ where $e^{2}=e \in \Gamma(p)$; therefore $e(A)=A, p(A)=A$.

There is a two-sided consequence of Corollary 1 given in [12], namely: if $A$ is a compact subset of $S$, and if $p, q \in S$ with $\Gamma(p)$ and $\Gamma(q)$ compact, then $p A q \supset A$ implies $p A q=A$.

The following corollary extends a result of the author [7].

Corollary 2. Let $S$ be compact, $a \in S$, and $a \in S a S$; then $S a$ is maximal among $\{S b \mid b \in S a S\}$.

Proof. Suppose $b \in S a S$ and $S a \subset S b$; then $b=x a y$ for suitable $x, y \in S$. Hence $S a \subset S b=S x a y \subset S a y$, so by Corollary $1, S a=S a y$ and $S a=S b$.

We remark that under the same hypotheses it can also be shown that $a S a$ is maximal among $\{b S c \mid b, c \in S a S\}$. The proof makes use of the remarks following Corollary 1.

The next two theorems are the analogs for semigroups of Gottschalk's extensions [4] of Weil's theorem [13] on monothetic groups. The proofs can be based on Gottschalk's results, but we prefer to give them in the language of semigroups.

Lemma 1. Let $S$ be locally compact with a compact kernel $K$; then there are arbitrarily small open sets containing $K$ which are ideals in their 
closures (i.e., for any open $V$ containing $K$, there is an open set $J$ with $K \subset J \subset V$ and $\bar{J} J \cup J \bar{J} \subset J)$.

Proof. Let $V$ be an open set containing $K$ and having compact closure. Note that $K \bar{V}=K \subset V$, so we may find an open set $W$ with $K \subset W \subset V$ and $W \bar{V} \subset V$ (this is conveniently done by using a lemma of Wallace [11]). Since $W \subset V$, we have $W^{2} \subset V, W^{3} \subset V, \cdots$, $\cup_{n} W^{n} \subset V$. Denote $\left[\cup_{n} W^{n}\right]^{*}$ by $P$, and observe that $P$ is a compact semigroup. We let $J$ denote the largest ideal of $P$ contained in $W$; we claim $J$ is open. Let $x \in J$; then $x \cup(P x) \cup(x P) \cup(P x P) \subset J \subset W$. Since $P$ is compact and $W$ is open, we may find an open set $N$ about $x$ such that $N \cup P N \cup N P \cup P N P \subset W$. But this latter set is an ideal of $P$, hence is contained in $J$. Hence $x \in N \subset J$, and the claim is established. Since $J$ is an ideal of $P$, it is certainly an ideal in $\bar{J}$, completing the proof.

The preceding lemma extends a result of Numakura [9].

THEOREM 4. Let $S$ be locally compact and monothetic, and suppose $S$ has a kernel $K$; then $S$ is compact.

Proof. Since $S$ is commutative it follows that $K$ is the unique minimal left and the unique minimal right ideal, hence is a group. Now $K=S e$ where $e$ is the unit of $K$, so $K$ is a retract of $S$, hence closed and locally compact. Also $K$ is monothetic $(+)$ with generator $a e$ where $a$ is a generator of $S$, hence $K$ is monothetic $( \pm)$. Since $K$ is locally compact and separable it follows that inversion is continuous (this fact is implicit in [2] and was observed by Neal J. Rothman in his Tulane Master's thesis, unpublished). By Weil's lemma $K$ is either compact or a copy of the group of integers. But the group of integers is not monothetic (+), hence $K$ is compact. By Lemma 1 we may find an open semigroup with compact closure $J$ containing $K$. Some power $a^{k}$ of the generator lies in $J$, hence $\Gamma\left(a^{k}\right) \subset \bar{J}$ and $\Gamma\left(a^{k}\right)$ is compact. Now let $M=\left\{a^{m}\right\}_{m=1}^{k-1}$; then $S=\Gamma(a)=M \cup M \cdot \Gamma\left(a^{k}\right)$, hence $S$ is compact.

Lemma 2. Let $S$ be locally compact with unit 1, and suppose the component $C$ containing 1 is compact. Then any open set about $C$ contains an open and compact semigroup about $C$.

Proof. Let $V$ be an open set containing $C$; we may find $W$ open and compact with $C \subset W \subset V$. Now let $P=\{t \mid t W \subset W\}$ and note that $P$ is a semigroup containing $1, P \subset W$, and since $W$ is both open and compact, so is $P$. Since $C$ is connected, $C \subset P$.

Lemma 2 and its proof are due to A. D. Wallace. 
THEOREM 5. Let $S$ be locally compact and monothetic with unit 1, and suppose the component $C$ containing 1 is compact; then $S$ is a compact group.

Proof. Let $P$ be a compact open semigroup containing $C$. Since $P$ is open, there is some power $a^{k}$ of the generator inside $P$. Since $P$ is compact so is $\Gamma\left(a^{k}\right)$ and it follows that $S(=\Gamma(a))$ is a compact group.

If $S$ is locally compact, monothetic, and has a unit, it is unknown whether $S$ must be compact. Under these hypotheses the conclusion is equivalent to: $S$ is a group. Another way of phrasing the question is this: if $S$ satisfies the above hypotheses, does there exist in $S$ an open semigroup with compact closure?

\section{REFERENCES}

1. A. H. Clifford, Semigroups containing minimal ideals, Amer. J. Math. vol 70 (1948) pp. 521-526.

2. Robert Ellis, Continuity and homeomorphism groups, Proc. Amer. Math. Soc. vol. 4 (1953) pp. 969-973.

3. B. Gelbaum, G. K. Kalisch and J. M. H. Olmsted, On the embedding of topological semigroups and integral domains, Proc. Amer. Math. Soc. vol. 2 (1951) pp. 807-821.

4. W. H. Gottschalk, Almost periodic points with respect to transformation semigroups, Ann. of Math. vol. 47 (1946) pp. 762-766.

5. P. R. Halmos and H. Samelson, On monothetic groups, Proc. Nat. Acad. Sci. U.S.A. vol. 28 (1942) pp. 254-258.

6. J. L. Kelley, Convergence in topology, Duke Math. J. vol. 17 (1950) pp. 277-283.

7. R. J. Koch, Remarks on primitive idempotents in compact semigroups with zero, Proc. Amer. Math. Soc. vol. 5 (1954) pp. 828-833.

8. K. Numakura, On bicompact semigroups, Mathematical Journal of Okayama University vol. 1 (1952) pp. 99-108.

9. - On bicompact semigroups with zero, Bulletin of the Yamagata University (natural science) no. 4 (1951) pp. 405-411.

10. J. E. L. Peck, An ergodic theorem for a noncommutative semigroup of linear operators, Proc. Amer. Math. Soc. vol. 2 (1951) pp. 414-421.

11. A. D. Wallace, $A$ note on mobs, Anais da Academia Brasileira de Ciencias vol. 24 (1952) pp. 329-334.

12. - Inverses in Euclidean mobs, Mathematical Journal of Okayama University vol. 3 (1953) pp. 23-28.

13. A. Weil, L'intégration dans les groupes topologiques et ses applications, Actualités Scientifiques No. 869, Paris, Hermann, 1938.

Louisiana State University 